Revista de Estudios Histórico-Jurídicos

[Sección derecho romano]

XXXVII (Valparaíso, Chile, 2015)

[pp. 77 - 92]

\title{
EL DECLIVE DE LAS CLASES MEDIAS Y LA CRISIS INSTITUCIONAL DE LA REPÚBLICA TARDÍA
}

[The Decline of the Middle Class and the Institutional Crisis of the Late Republic]

\author{
Carlos Felipe Amunátegui Perelló* \\ Pontificia Universidad Católica de Chile, Santiago de Chile
}

\begin{abstract}
RESUMEN
Los regímenes políticos participativos requieren la presencia de una clase media que les sirva de base social. Tanto en el caso de la República romana, como en las democracias modernas, su desarrollo armónico ha sido consecuencia de la existencia de un amplio segmento medio de la sociedad. En Roma, el declinar de este segmento medio terminó en una crisis de gobernanza que llevó al fin de la República.

\section{Palabras Clave}

Clase romana media - Distribución de la riqueza en Roma.
\end{abstract}

\begin{abstract}
Participatory political regimes require a middle class social basis. Both in the case of the Roman Republic as well as in modern democracies, its harmonic development has been the result of the presence of a wide middle class segment in the society. When this segment declined in Rome, a crisis in governance took place and led to the end of the Republic.

Keywords

Roman middle class - Distribution of wealth in Rome.
\end{abstract}

RECibIDO el 18 de mayo y ACEPTADO el 1 de julio de 2015

* Profesor de derecho romano, en la Pontifica Universidad Católica de Chile. Dirección postal: Facultad de Derecho, Pontificia Universidad Católica de Chile, Avenida Bernardo O’Higgins 340, Santiago, Chile. Correo electrónico: camunate@uc.cl El presente artículo es parte del Proyecto FONDECYT regular No 1141231 y del Proyecto Anillos de Investigación SOC 1111. 


\section{INTRODUCCIÓN}

La aquiescencia de los ciudadanos es un requisito fundamental para que cualquier sistema político llegue a funcionar. En efecto, todos los sistemas políticos necesitan de la colaboración de los destinatarios del poder, aunque aquéllos que son percibidos como injustos -que carecen de legitimidad-requerirán de una mayor coacción del aparato estatal para su funcionamiento, pero todos, en cierta medida, alternan el uso de la fuerza con la aceptación voluntaria de los propios súbditos del estado de cosas imperante. La proporción de fuerza que un sistema requiere es mayor en la medida que sus resultados sean percibidos como más injustos, y decrece naturalmente en aquellos sistemas donde los ciudadanos se sienten partícipes de los éxitos del mismo. Mientras mayor sea la percepción de injusticia, más deberá invertir la elite en proporcionar medios coercitivos que mantengan el sistema político en orden y, por tanto, menor será la capacidad del mismo para expandirse y desarrollarse. En palabras de North "The costs of maintenance of an existing order are inversely related to the perceived legitimacy of the existing system"'.

En los sistemas políticos participativos, como la democracia moderna, la aceptación por parte de los propios ciudadanos del estado de cosas imperante resulta fundamental, toda vez que su participación activa es indispensable para su funcionamiento, aunque sea sólo para convencerlos de ir a las urnas cada cierta cantidad de años.

No es una casualidad que la democracia se estabilizara en Europa luego de las guerras mundiales, que desataron uno de los procesos más masivos de equiparación económica que la humanidad ha conocido ${ }^{2}$. Las fortunas de las antiguas elites fueron fulminadas por la inflación aparejada al abandono del patrón oro, la destrucción física del capital proveniente de la guerra y los vaivenes bursátiles de la Gran Depresión. Esta brutal equiparación trajo como consecuencia que la herencia, como fuente de riqueza, decayese ${ }^{3}$ frente a las posibilidades que se abrían al hombre común con tal que tuviese acceso a una educación. El colapso de la concentración del capital durante el período se tradujo en la creación de un sistema social percibido como equitativo, donde la educación y el trabajo eran considerados como presupuestos de la riqueza.

El presente es un ejercicio comparativo que intentará tomar el caso de otra sociedad, la Roma republicana, donde un proceso de acumulación de riqueza descontrolado terminó por sepultar un sistema político participativo dando lugar al nacimiento del Principado.

${ }^{1}$ North, Douglas C., Structure and Change in Economic History (New York, Norton, 1981), p. 53.

${ }^{2}$ PiketTy, Thomas, Capital in the Twenty-First Century (Cambridge, Harvard University Press, 2014), pp. 126-139, entrega datos interesantes relativos a la distribución del ingreso en Inglaterra y Francia durante los siglos XIX y XX. Lo más llamativo del fenómeno es la enorme igualación en la distribución que tuvo lugar luego de las guerras mundiales y la Gran Depresión.

${ }^{3}$ Piketty, Thomas, Capital in the Twenty-First Century (n. 2), p. 377. 


\section{LA CONCENTRACIÓN DE LA RIQUEZA Y LA CLASE MEDIA EN ROMA REPUBLICANA}

Antes de estudiar los problemas de gobernanza que se suscitan en la Antigüedad Clásica, es menester considerar algunos puntos clave relativos a la generación y distribución de la riqueza que se suscitan, en general, en todas las sociedades preindustriales. En primer término, debe tenerse en cuenta que en dichas sociedades crecimiento económico es prácticamente nulo, ante lo cual, cualquier aumento de la población genera, necesariamente, una nueva provisión de desposeídos cuyo destino vital es incierto ${ }^{4}$. En efecto, la Antigüedad era un mundo malthusiano, donde los aumentos de productividad generados por las pocas innovaciones técnicas que se implementan en los medios de producción, rápidamente son absorbidas por aumentos de población que conducirán a una nueva crisis social ${ }^{5}$. En tales condiciones, la forma más simple de aumentar el producto geográfico bruto es incrementar la masa de capital fijo mediante la conquista militar de los territorios vecinos ${ }^{6}$. De ahí que la guerra tuviese un rol central en las sociedades antiguas, puesto que el éxito militar significaba lo mismo que para las sociedades industriales el crecimiento económico. La obsesión militar era especialmente importante para aquellas sociedades que enfrentaban un crecimiento demográfico importante o que tuviesen un gobierno participativo preocupado por la mejora efectiva de las condiciones de vida de sus ciudadanos. Por ello, las democracias de la Antigüedad resultan invariablemente imperialistas, mientras que las aristocracias no necesariamente.

Otro factor a considerar es que la estructura económica de la Antigüedad, en general, era poco proclive hacia el trabajo remunerado. Aunque éste indudablemente existía ${ }^{7}$, su rol económico era marginal, sobre todo ante la presencia ubicua de la esclavitud ${ }^{8}$, como también de otras formas de asegurar el trabajo subordinado

${ }^{4}$ Harris, William V, Poverty and Destitution in the Roman Empire, en Rome's Imperial Economy, Twelve Essays (Oxford - New York, Oxford University Press - Kindle, 2011), p. 1.349.

${ }^{5}$ Acerca del crecimiento económico en la Antigüedad, el debate parece centrarse hoy en día en la velocidad con que los aumentos de población son capaces de absorber el crecimiento económico. Al respecto, véanse: SCHEIDEL, Walter, Demography, en ScHEIDEL, Walter - MorRIs, Ian - SAller, Richard (editores), The Cambridge Economic History of the Greco-Roman World (Cambridge, Cambridge University Press, 2007), pp. 321-333; SALLE, Richard, Framing the Debate over Growth in the Ancient Economy, en SCHEIDEL, Walter - VON REDEN, Sitta (editores), The Ancient Economy (New York, Routledge, 2010) pp. 251-269; Temin, Peter, The Roman Market Economy (New Jersey, Princeton University Press, 2013), pp. 195-239.

${ }^{6}$ En este sentido señala Morley, Neville, Trade in Classical Antiquity (Cambridge, Cambridge University Press-Kindle Ed., 2007), p. 1.505 que "the importance of violence as a mode of accumulation and a cultural practice in antiquity can scarcely be exaggerated".

${ }^{7}$ Recientemente, el rol del trabajo remunerado en la Antigüedad se encuentra siendo reexaminado. Se ha planteado la posibilidad de la existencia de un verdadero mercado de trabajo: véanse: Harris, William V, Poverty and Destitution in the Roman Empire, cit. (n. 4), pp. 1.5461.578; Kenoe, Denis, Contract labor, en Scheidel, Walter (editor), Roman Economy (New York, Cambridge University Press, 2012), pp. 114-131. La posición más extrema en la materia apunta a la existencia de un mercado de trabajo plenamente operativo y amplio para Roma Imperial, como se ve en Temin, Peter, The Roman Market Economy, cit. (n. 5), pp.114-138).

${ }^{8} \mathrm{La}$ esclavitud parece haber sido introducida durante la monarquía etrusca, aunque natural- 
aplicadas en épocas tempranas ${ }^{9}$. Ello hacía que, a diferencia de nuestra sociedad ${ }^{10}$, la distribución del ingreso y del capital fuesen prácticamente equivalentes. El irrelevante crecimiento económico y la concentración de los ingresos en el capital hacen que las crisis de gobernanza en la Antigüedad se tradujesen rápidamente en demandas de reforma agraria, especialmente respecto a los territorios recientemente conquistados.

\section{La Repartición DE la tierra y las “leges Liciniae SeXtiae”}

De acuerdo a la historiografía romana, los comienzos de la República fueron agitados, socialmente hablando. La tradición reporta una larga serie de conflictos entre dos grupos sociales, los patricios y los plebeyos, cuyos orígenes no parecen del todo claros ${ }^{11}$. Este conflicto se habría desenvuelto por tres materias clave: el

mente no puede haber certeza acerca de ello. Véase: Franciosi, Gennaro, "Res mancipi" e "res nec mancipi", en Labeo, 5-3 (1959), p. 375; Franciosi, Gennaro, Famiglia e persone in Roma antica (Torino, Giappichelli, 1992), pp. 206; DE MarTino, Francesco, Clienti e condizioni materiali in Roma arcaica, en Diritto economia e società nel mondo romano (Napoli, Jovene, 1997), pp. 82-83; De Martino, Francesco, Intorno all origine della schiavitù a Roma, en Diritto economía e società nel mondo romano (Napoli, Jovene, 1997), pp. 27-57.

${ }^{9}$ Entre ellas mencionaremos la clientela, con su obligación de prestar servicios al patrono, el nexum, como préstamo garantizado con la persona del deudor, y la transferencia de los hijos a través del mancipium.

${ }^{10}$ En efecto, en el mundo moderno la distribución del ingreso y del capital son distintos. En general, el capital tiende a estar mucho más concentrado que los ingresos, toda vez que entre estos últimos se cuentan también las rentas del trabajo. Véase PIKETTY, Thomas, Capital in the Twenty-First Century, cit. (n. 2), pp. 39-71.

${ }^{11}$ Las teorías respecto al origen de los grupos patricio y plebeyo son variadísimas. Algunas parten del supuesto dualista donde habría una diferenciación étnica entre ambos grupos, donde los patricios serían etruscos y los plebeyos latinos; al respecto, véase: ArANGIO-RuIZ, Vincenzo, Storia del diritto romano (Napoli, Jovene, 2006), pp. 21); o al menos respecto a su condición de ciudadanos, véase: GuarIno, Antonio, Le origine quiritarie. Raccolta di Scritti romanistici (Napoli, Jovene, 1973), pp. 9-17. Otros prefirieron establecer una evolución donde el grupo patricio y plebeyo se forman a lo largo de la monarquía, sea por el ejercicio de funciones sacerdotales, como piensa Mitchell, Richard E., The Definition of patres and plebs: An End to the Struggle of the Orders, en RAAFLAUB, Kurt, Social Struggles in Archaic Rome. New Prespectives on the Conflict of the Orders (Oxford, Blackwell, 2005), pp. 128-167, o apartir de la acumulación de riqueza y el ejercicio del poder en el Senado, según HaRmand, Louis, Société et économie de la république romaine (Paris, Armand Colin, 1976), p. 32; Drummond, Andrew, Rome in the Fifth Century II: The Citizen Community, en Walbank, F. W. - Astin, A.E. - Frederiksen, M.W. - Ogilvie, R.M. (editores), The Cambridge Ancient History (Cambridge, Cambridge University Press, 1989), VII, p. 2 y 172-242; Momigliano, Arnaldo, The Origins of Rome, en The Cambridge Ancient History, cit. ibi, VII, pp. 52-112; y Momigliano, Arnaldo, The Rise of the plebs in the Archaic Age of Rome, en RaAflaub, Kurt, Social Struggles in Archaic Rome. New Prespectives on the Conflict of the Orders (Oxford, Blackwell, 2005), pp. 168-184; Magdelain, André, De L'auctoritas patrum à lauctoritas senatus, en Jus imperium auctoritas. Études de droit romain (Paris, Ecole Française de Rome, 1990), pp. 385-403; Cornell, Tim J., The Beginnings of Rome and Italy from the Bronze Age to the Punic Wars (c.1000-264 BC) (London - New York, Routledge, 1995), pp. 244-258; RaAflaub, Kurt, From Protection and Defense to Offense and Participation: Stages in the Conflict of the Orders, en RaAflaub, Kurt (editor), Social Struggles in Archaic Rome. New Prespectives on the Conflict of the Orders (Oxford, Blackwell, 2005), pp. 
conocimiento del Derecho, el acceso a las magistraturas y el control de la tierra pública. Aunque el debate académico respecto a este conflicto se ha prolongado literalmente por siglos y ha alcanzado ocasionalmente niveles de complejidad altísimos, nosotros nos centraremos exclusivamente en el problema de la tierra y su distribución.

Parte de la doctrina estima que todo el conflicto es una mera invención, una especie de anticipación pseudo histórica de los verdaderos conflictos por el control de la tierra que sucedieron durante el siglo II a. C. en adelante ${ }^{12}$, aunque hay bastantes elementos como para descartar en todo o parte esta tesis y estimar que la lucha por la tierra es efectivamente un elemento central del conflicto patricio-plebeyo ${ }^{13}$.

Aparentemente, el grupo denominado plebeyo no tenía acceso al control de las tierras recién conquistadas, las cuales quedaban en calidad de tierras públicas (ager publicum) a disposición de la población para ser ocupadas ${ }^{14}$. Desconocemos las razones exactas de por qué, en la práctica, sólo los patricios tenían acceso a ellas ${ }^{15}$ y eran ellos los únicos que las ocupaban efectivamente, pero todo el conflicto

185-222; Forsythe, Gary, A Critical History of Early Rome (Berkeley -Los Angeles, California University Press, 2006), p. 162; SMITH, Christopher, The Roman Clan. The gens from Ancient Ideology to Modern Anthropology (Cambridge, Cambridge University Press, 2006), pp. 305); o por la inmigración de artesanos de vocación predominantemente urbana (plebeyos), frente a una aristocracia tradicionalmente agrícola (patricios), como en RICHARD, Jean-Claude, Réflexions sur les origines de la plebe, en Bilancio critico su Roma arcaica fra monarchia e repubblica (Roma, Academia Nazionale dei Lincei, 1993), pp. 27-41; y Richard, Jean-Claude, Patricians and Plebeians: The Origin of a Social Dichotomy, en RAAFLAUB, Kurt (editor), Social Struggles in Archaic Rome. New Prespectives on the Conflict of the Orders (Oxford: Blackwell, 2005), pp.107127), para terminar con el cierre del patriciado a comienzos de la República. La postura que nos parece más probable es, en todo caso, aquélla que estima que los patricios son los miembros de los antiguos clanes gentilicios, frente a los plebeyos que serían ajenos a ellos, según BonfanTE, Pietro, La gens e la familia, en Scritti giuridici. Famiglia e successione. (Torino, Unione Tipografico, 1916), pp. 1-17; Bonfante, Pietro, Teorie vecchie e nuove sulle formazione sociali primitive, en Scritti giuridici. Famiglia e successione (Torino, Unione Tipografico, 1916) pp. 18-63; Castello, Carlo, Studi suldiritto familiar e gentilizio romano (Roma, L'Erma di Bretschneider, 1942), p. 49; De Martino, Francesco, La gens, lo stato e le classi in Roma antica, en Diritto economía e società nel mondo romano (Napoli, Jovene, 1997), pp. 25-49; DE MARTino, Francesco, Storia costituzionale romana (Napoli, Jovene, 1973) p. 78; CAPOgrossi Colognesi, Luigi, Diritto e potere nella storia di Roma (Napoli, Jovene, 2007), pp.49-51.

${ }^{12}$ MASCHKE, Richard, Zur Theorie und Geschichte der römischen Agrargesetze (Napoli, Jovene, 1980), p. 14; GaBBA, Emilio, Motivazioni economiche nell opposizione alla lege agraria di Tib. Sempronio Graccho, en Evans, J.A.S. (editor), Polis and Imperium: Studies in Honour of Edward Togo Salmon (Toronto, Hakkert, 1974), p.135.

${ }^{13}$ Serrao, Feliciano, Lotte per la terra e per la casa a Roma, en SERrao, Feliciano (editor), Legge e società nella repubblica romana (Napoli, Jovene, 1981) p. 67; DRUMMOND, Andrew, Rome in the Fifth Century II, cit. (n. 11), p. 237; y RoselaAr, Saskia T. Public Land in the Roman Republic. A Social and Economic History of Ager Publicus in Italy, 396-89 BC (Oxford - New York, Oxford, 2010), p. 28.

${ }^{14}$ Sicul. Flacc., de condic. agr. 101,9-13 y 102,9-13.

${ }^{15}$ En efecto, la exclusividad del control patricio de las tierras conquistadas y en régimen de ager publicus es un hecho que se desprende de la tradición. Al respecto Nonnio reporta que por su condición de plebeyos son expulsados del ager publicus (Non. 149 M.17: "Quicumque propter 
patricio-plebeyo giró, durante la República temprana, en torno a la constante demanda plebeya por su distribución. Los efectos disruptivos de las leyes de reforma agraria quedan clásicamente expresados en la observación de Livio acerca de que estas nunca se ejercen sin los mayores desórdenes públicos ${ }^{16}$.

Sea como fuese, el conflicto patricio-plebeyo finalmente baja de intensidad sólo en el 367, luego de 140 años de permanentes hostilidades, con las leges LicianaeSextiae que, entre otras cosas, limitan a un máximo individual la posibilidad de ocupar el ager publicus. De aquí en adelante, cada conquista militar acarreará una distribución agraria, sea en forma de asignación directa (viritaria) o del establecimiento de una colonia de ciudadanos en el territorio confiscado a los enemigos ${ }^{17}$. Así describe Apiano el proceso ${ }^{18}$ : "Los romanos, a según sometían por la guerra distintas regiones de Italia, tomaban parte de su territorio y establecían ciudades, o reclutaban colonos propios para enviarlos a las que ya habían”.

Resulta interesante corroborar la tradición con la información que la arqueología ofrece. Al respecto, a contar del siglo IV a. C. se observa una verdadera explosión de asentamientos de modestas dimensiones que se corresponden con la parcelación de tierras y su ocupación por pequeños agricultores, proceso que puede ser ligado a las leyes Licianiae Sextiae en Italia Central ${ }^{19}$.

Esta política de asignación constante de nuevos territorios a los desposeídos resulta clave a la hora de explicarse la buena gobernanza y el impulso militar que Roma tuvo a lo largo de la Alta República. La ciudad estaba socialmente bien cohesionada, toda vez que los beneficios de su principal actividad pública -la

plebitatem agro publico eiecti sunt". Las razones nos son ignotas, pero bien puede deberse a su falta de vinculación con los clanes (gentes) originales que componen la ciudad, o simplemente a su menor poder social. Para la época monárquica, la tradición reporta varias distribuciones de territorio realizadas por los reyes a favor de los desposeídos (Dion Hal. 2,62,2-3; 3, 1,4-5; 2,29,6; $3,31,3 ; 3,9,8 ; 4,10,3 ; 4,13,1)$, pero llegados a la República temprana estas asignaciones cesan y el territorio conquistado queda siempre bajo el régimen de ager publicus, salvo para los casos en que la tradición reporta excepcionalmente que se crea alguna colonia (Liv. 2,21,6; 2,31,4; 2,34,6; 3,1,5-6), hasta la distribución del Aventino entre la plebe por la lex Icilia del 456. En cambio, las demandas de la plebe por una distribución del terreno público son frecuentes y casi anuales, contándose más de diez proyectos rechazados por los patres entre el 486 y el 456; véanse: Capogrossi Colognesi, Luigi, La terra in Roma antica (Roma, La Sapienza, 1981), p. 6; Manzo, Annamaria, La lex licinia sextia de modo agrorum (Napoli, Jovene, 2001), pp. 4045; Russo, Adelaide, Tiberio Gracco e la riforma agraria, en La romanizzazione della Campania antica (Napoli, Jovene, 2002), pp. 161-193; SмITH, Christopher, J., The Roman Clan, cit. (n. 11), p. 240; y Roselaar, Saskia, Public Land in the Roman Republic, cit. (n.13), pp. 26-29).

${ }^{16}$ Liv. 2,41,3: "lex agraria promulgata est, nunquam deinde usque ad hanc memoriam sine maximis motibus rerum agitate".

${ }^{17}$ No se sabe a ciencia cierta cuánto territorio se confiscaba al enemigo luego de una conquista militar. Usualmente se estima que un tercio de su territorio pasaba a ser controlado por Roma, aunque hay casos en que esta cifra crecía a la mitad e incluso dos tercios. Al respecto: Roselaar, Saskia, Public Land in the Roman Republic, cit. (n. 13), pp. 31-37.

${ }^{18}$ App. BC 1,1,7: "Rwmaiĺoi th $\backslash n$ ' ${ }^{1}$ tali roj e)la/mbanon kaì̀ po/leij e)n\%"kizon hÄ e)j talj pro/teron ouÂsaj klhrou/xouj a)pol sfw1/2n kate/legon".

19 Terrenato, Nicola, The essential Countryside, en Alcock, Susan - Osborne, Robin, Classical Archeology (Malden - Oxford, Blackwell, 2012), pp. 147-148. 
guerra- recaían en el conjunto de sus ciudadanos y, especialmente, en los más pobres. La guerra se tornó una actividad popular, toda vez que constituía una fuente inagotable de beneficios económicos para los ciudadanos y cimentaba la estabilidad económico-social de la ciudad. El éxito militar equivalía, por la entrada masiva de capital fijo, al aumento en el producto geográfico bruto y, por consiguiente, al crecimiento económico. En palabras de Harris, Roma exportó sus pobres al resto de Italia y luego a las provincias ${ }^{20}$. En total, cerca de setenta mil hombres, con sus familias, fueron provistos de tierras sólo durante las sucesivas guerras samnitas ${ }^{21}$.

\section{LA CLASE MEDIA EN LAS GUERRAS ANIBÁLICAS}

Los beneficios de la guerra equivalían al crecimiento del PGB. Este crecimiento era distribuido entre los ciudadanos directamente en virtud de la repartición de tierras, lo cual cimentó la creación de lo que podríamos llamar una clase media romana. Aunque el concepto de clase media es, naturalmente, exótico para la realidad social romana, podemos, siguiendo a Harris ${ }^{22}$, clasificar a la población de acuerdo a un criterio altamente flexible, tomado en último término de Aristóteles, entre aquéllos que pueden vivir confiando en el trabajo ajeno de esclavos y dependientes, aquéllos que tienen suficiente tierra como para asegurarse su subsistencia familiar a través de su propio trabajo y los que son trabajadores por cuenta ajena, sean libres o esclavos ${ }^{23}$. Es en este sentido que los beneficiarios de la política agraria distributiva de la Roma republicana pueden ser concebidos como la clase media.

Naturalmente no podemos cuantificar directamente su importancia dentro de la estructura social romana, sobre todo porque no contamos con cifras estadísticas exactas respecto a la pobreza, la riqueza o siquiera la cantidad de esclavos que existía en Roma en ningún momento. No obstante, existe un dato relativamente confiable que, en cierta medida, nos puede ayudar a estimar, aunque sea de manera aproximada, su extensión. Este es el número de legiones activas en Roma.

El ejército republicano romano era reclutado en base al patrimonio y exclusivamente entre las personas que tenían medios de vida, excluyéndose de él, hasta la reforma de Mario, a los proletarios. Al respecto, contamos con información bastante precisa gracias al testimonio de $\mathrm{Livio}^{24}$ que, aunque puede resultar ana-

${ }^{20}$ Harris, William, Poverty and Destitution in the Roman Empire, cit. (n. 4), p. 1.439.

${ }^{21}$ Cornell, Tim, The Conquest of Italy, en Walbank, F. W. - Astin, A. E. - Frederiksen, M. W. - Ogilvie, R. M. (editores), The Cambridge Ancient History (Cambridge, Cambridge University Press, 1989), VII, p. 405

${ }^{22}$ Harris, William, Poverty and Destitution in the Roman Empire, cit. (n. 4), pp. 1.308-315.

${ }^{23}$ Siguiendo un criterio similar, KNAPP, Robert, Invisible Romans (Cambridge, Harvard University Press, 2011) p. 5, habla del “ordinary man” en Roma, definiéndolo como aquel que se encuentra bajo la elite de senadores y caballeros, pero por sobre el jornalero y el esclavo.

${ }^{24}$ Liv. 1,43. El pasaje describe la reforma del rey Servio Tulio que introduciría la estructura de las centurias como unidad básica de organización militar y política en Roma. Las cifras ahí aportadas son increíbles para la Roma monárquica, puesto que implicarían la existencia de un ejército de casi veinte mil hombres para una ciudad que a lo sumo tendría unos $50 \mathrm{mil}$. La hipótesis más probable es que dicha descripción esté basada en una reforma realizada en torno 
crónica para la Roma de los reyes, parece, hasta cierto punto, confiable para la organización de Roma republicana. En ella se describen cinco clases de las cuales se recluta al ejército en razón de su patrimonio, mientras que aquellos que quedan bajo este nivel económico se encuentran en la infraclase de los proletarios. Es probable que después de la introducción del pago por el servicio en el ejército en el año 406 a. C. las legiones se reclutasen indistintamente de todas las clases, con tal que estuviesen sobre el mínimo de once mil ases establecido para los proletarios ${ }^{25}$.

Durante la Segunda Guerra Púnica Roma llevó su capacidad militar hasta sus últimas consecuencias levantando un promedio de veinticinco legiones durante los veinte años de conflicto. Cada legión, según Polibio ${ }^{26}$, contaría con unos cuatro mil doscientos hombres, aunque la cifra definitiva puede ser algo superior, puesto que las legiones podían llegar a tener hasta 6 mil hombres. Si tomamos como media cinco mil hombres por legión, la clase de hombres dotados de un patrimonio suficiente como para hacer frente a las obligaciones militares sumaría unos $125 \mathrm{mil}$ hombres. Si tenemos en consideración que el porcentaje de hombres adultos en las ciudades de la Antigüedad suele situarse en torno al 25\% de la población ${ }^{27}$, dicha cifra supone una población dotada de un estándar de vida medio de unas 500 mil personas. Aunque la población total de Italia debió ser de aproximadamente tres millones de habitantes ${ }^{28}$, la población romana poco después del fin de las guerras anibálicas fue censada en 243.704 ciudadanos varones ${ }^{29}$. Si suponemos que los hombres representan aproximadamente la mitad de las mujeres (aunque en un período de desastres militares la cifra puede ser bastante inferior), la población total de ciudadanos romanos sería de unas 480 mil personas adultas, a los que habría que agregar a los menores de 14 años, que no suelen ser censados. De acuerdo a las tablas de población utilizadas por $\mathrm{Saller}^{30}$, si usamos el límite inferior de las mismas, es decir, una esperanza de vida de 25 años, la cifra supondría un 50\% de la población (dado que la mortandad infantil entre los 0 y 10 años alcanzaría a casi la mitad de los nacidos), mientras que si usamos el límite superior (32,5 años), dicho total alcanzaría al $60 \%$ de la población. Es decir, la cifra del censo implicaría una población total, incluyendo niños, entre 800 y 960 mil ciudadanos.

al año 241 a. C. Véase: Cornell, Tim, The Beginnings of Rome, cit. (n. 11), p. 180; SMITH, Christopher, The Roman Clan, cit. (n. 11), p. 174; ForsyTHE, Gary, A Critical History of Early Rome, cit. (n. 11), p. 113.

${ }^{25}$ Según Polib., Hist. 6,19 (8), serían 400 dracmas en el año 216 a. C.

${ }^{26}$ Polib., Hist. 6,20,8.

${ }^{27}$ En este sentido, véase: RaAflaub, Kurt A., From Protection and Defense to Offense and Participation, cit. (n. 11), p. 22. Un estudio más detallado basado en esperanzas de vida más altas y más bajas fue realizado recientemente fijando, para la esperanza de vida más baja en 25 años y la más alta en 32,5 años por SCHeidel, Walter, Demography, cit. (n. 5) p. 40.

${ }^{28}$ Cornell, Tim, The Conquest of Italy, cit. (n. 21), p. 405; SCHeidel, Walter, Demography, cit. (n. 5), p. 45.

${ }^{29}$ Liv. 35,9,21,1. Los censos de la República media usualmente son estimados confiables, véase: Lo Cascio, Elio, Population and Demographic Studies, en Derose, Jane Evans (editora), A Companion to the Archeology of the Roman Republic (Oxford, Blackwell, 2013,) pp. 155-166.

${ }^{30}$ SAller, Richard, Patriarchy, Property and Death in the Roman Family (Cambridge, Cambridge University Press, 1994), p. 25. 
En resumidas cuentas, aunque las cifras que entregamos no pueden ser exactas y simplemente intentan dar órdenes de magnitud, parecen apuntar a que entre un 52 y un $62 \%$ de la población disponía de suficiente propiedad como para hacer frente a las necesidades diarias de la vida ${ }^{31}$.

Esto supone una cifra relativamente alta para lo que denominaríamos clase media romana. Es cierto que en esta cifra estamos ignorando deliberadamente la situación de los esclavos y de los extranjeros residentes en territorio romano, así como también de los demás habitantes de Italia que perteneciesen a otras comunidades ciudadanas y que, a pesar de estar sometidos a Roma, no participan de su sistema político. No obstante, la cifra es elocuente. La mayoría de los ciudadanos romanos tenían una posición económica que les permitía vivir de manera independiente, trabajando sus propios terrenos, y sirviendo en el ejército durante las Guerras Anibálicas.

Respecto a la elite, no tenemos ninguna forma de cuantificarla, ni de medir los recursos que absorbía, pero siempre fue un grupo bastante reducido. El total de senadores no superaba los trescientos y si suponemos un grupo diez veces mayor de caballeros y les agregamos sus familias, el total no pudo superar las doce mil personas, una cifra que está dentro del orden del $1 \%$ de la población total. En pocas palabras, y volviendo a las tres categorías sociales apuntadas por Harris, podríamos pensar en una población romana dividida entre una elite que fluctúa entorno al $1 \%$ de la población, una clase media que cubre alrededor del $55-60 \%$ de la población y un proletariado que representa cerca del $40-45 \%$ de la población, cifras que no son muy diferentes de las que presenta una democracia avanzada hoy en día.

El sistema político republicano se cimentaba en esta clase media, que prestó al sistema político su ideal social de soldado, ciudadano y agricultor. Este arquetipo modélico sirvió como referente de la República, una suerte de encarnación ideológica de sus virtudes. Cincinato, que trabaja su pequeño lote de tierra con sus manos, llamado a salvar a la República por las armas y que devuelve la púrpura de la dictadura a los 15 días de asumido el cargo, es el prototipo del sueño republicano. El hombre medio, trabajador, soldado y ciudadano, puede verse en él reflejado y sentirse garante de la República.

Roma conquistó Italia impulsada por un sistema político armónico, donde sus ciudadanos eran los directos beneficiarios de la prosperidad obtenida y el apoyo a al ideal republicano era unánime. Con cierto exceso se ha descrito este período como la democracia romana ${ }^{32}$. Indudablemente se trata de un sistema político participativo, donde a través de los comicios y los tribunos, el hombre común

${ }^{31}$ Nuestro cálculo es levemente más optimista que el de BRUNT, Peter, Italian Manpower (London, Oxford University Press, 1971), pp. 64-66, que cifraba el total de assidui en torno al $50 \%$ de la población (pero significativamente más pesimista que la reciente estimación de Rosenstein, Nathan, Rome at War (Chapel Hill - London, North Carolina University Press, 2004), pp. 185-188, que cifra el número de proletarios durante las guerras anibálicas en torno al $10 \%$ de la población

${ }^{32}$ Guarino, Antonio, Forma e materia della costituzione romana, en Studi di diritto costituzionale romano (Napoli, Jovene, 2008), pp. 11-26. 
tiene una participación en la toma de decisiones y los resultados de las mismas revierten en su beneficio. Sus costos de mantención son extremadamente bajos, al punto que Roma no cuenta con una policía, que imponga el orden, ni con un aparato burocrático que administre sus éxitos. Llamarlo democracia o no es un mero problema semántico.

\section{REgResión DE LA CLASE MEDIA EN LA ROMA TARDO-REPUbLICANA}

Luego de las guerras anibálicas la situación social de Roma empeora rápidamente. El proceso ha sido descrito en numerosas ocasiones y puede relacionárselo con distintos fenómenos económico-sociales que el éxito militar de Roma implicó. El más importante de todos, desde nuestro punto de vista, fue la inserción de Roma en los mercados de grano de larga distancia que abastecían al Mediterráneo ${ }^{33}$.

La mayor parte de los pequeños agricultores romanos producían fundamentalmente trigo, aunque complementado con vides y olivos, marca característica de la coltora promiscua mediterránea que imperaba en Italia central desde el siglo VII a. C. ${ }^{34}$, a lo menos. Su foco fundamental era la satisfacción de sus propias necesidades y aunque la venta de su superávit en el mercado era un objetivo importante, a fin de poder obtener toda la amplia gama de productos que no eran capaces de producir por sí mismos. No obstante, la serie de conquistas realizadas por Roma puso a disposición del mercado local vastas cantidades de grano de calidad superior a la producida localmente de manera gratuita - en la forma de tributos- o a un precio módico, a consecuencia de la importación masiva de grano desde Egipto y África. Esto, inmediatamente, expulsó a los pequeños productores del mercado local, quienes evidentemente no podían competir. El resultado fue el abandono de los campos por parte de buena parte de la población rural, los que en buena medida quedaron desolados, incluso en las inmediaciones de Roma ${ }^{35}$, donde gozarían de bajos costos de transporte.

La integración de Roma en los mercados mediterráneos tuvo no sólo consecuencias negativas, sino que también presentó oportunidades para la elite que pudo beneficiarse de la expansión imperial. Los nuevos mercados presentaban amplias oportunidades para la exportación de productos agroindustriales, como el vino, la miel o el aceite. No obstante, para la mayor parte de los pequeños agricultores estas oportunidades estaban fuera de sus posibilidades. El pequeño agricultor produce una cosecha equilibrada para obtener un óptimo enfocado a satisfacer sus propias necesidades básicas de trigo. Alterar ese equilibrio a favor de un nuevo

\footnotetext{
${ }^{33}$ Esto es lo que Harris llama el "Hellenistic-Carthaginian system", que venía funcionando como mecanismo de distribución de bienes de este a oeste del Mediterráneo desde hacía siglos: Harris, William, The Late Republic", en Scheidel, Walter - Morris, Ian - Saller, Richard (editores), The Cambridge Economic History of the Greco-Roman World (Cambridge, Cambridge University Press, 2007), p. 51).

${ }^{34}$ Torelli, Mario, History: Land and People, en Bonfante, Larissa (editora), Etruscan Life and Afterlife (Detroit, Wayne State University Press, 1986), p. 52; ForsyTHE, Gary, A Critical History of Early Rome, cit. (n. 11), p. 56.

${ }^{35}$ Rosenstein, Nathan, Rome at War, cit. (n. 31), pp. 7.
} 
óptimo para la exportación de aceite o vino, supone exponerlo al hambre ${ }^{36}$, por lo que sus posibilidades de adaptarse a la nueva realidad son magras. Por lo demás, carecía del capital necesario para realizar esa reconversión ${ }^{37}$.

Las guerras conducidas fuera de Italia produjeron un inmenso flujo de liquidez producto de la destesaurización de las riquezas de los reinos helenísticos, cuyas amplias reservas de plata fueron amonedadas ${ }^{38}$. Este flujo revirtió en buena medida a favor de la elite republicana, que pudo adquirir, sea a través de la compra, sea a través de la violencia, los campos que eran al mismo tiempo abandonados por los pequeños agricultores. Con su recién adquirido capital pudieron establecer unidades productivas mayores dedicadas a la producción de superávits destinados a la exportación y trabajados mayoritariamente por mano de obra esclava. Estas son las villas romanas. Aparentemente, también la violencia fue utilizada para la obtención de algunas de las tierras de pequeños agricultores que fueron reconvertidas en villas. Apiano hace un vívido retrato de la situación ${ }^{39}$ : “Los ricos, acaparando la mayor parte de esta tierra no distribuida, aumentaron con el tiempo su confianza en que ya no se verían desposeídos de ella y, comprando en parte por métodos persuasivos, en parte apoderándose por la fuerza de las propiedades vecinas y de todas las demás pequeñas pertenecientes a campesinos humildes, cultivaban grandes latifundios [...]”. Y más adelante ${ }^{40}$ : "Por estas razones los ricos se enriquecían al máximo y los esclavos aumentaban muchísimo por la campiña; en tanto que la escasez y la falta de población afligían a los pueblos itálicos, diezmados por la pobreza, los tributos y la milicia”.

Aparte de los problemas relacionados con el mercado, la guerra, en sí misma, dejó de ser una fuente de bienestar para los ciudadanos. En efecto, los devastadores efectos de la ocupación de Italia durante casi veinte años por las tropas anibálicas han sido descritos como una de las causas más importantes de la decadencia del pequeño agricultor italiano ${ }^{41}$, aunque su efecto puede ser relativizado ${ }^{42}$. Un factor tal vez más importante es el desarrollo de conflictos bélicos de larga distancia y

\footnotetext{
${ }^{36}$ Roberts, Keith, Origins of Business, Money and Markets (New York, Columbia University Press - Kinlde, 2011), p. 1.274.

${ }^{37}$ No obstante, igualmente algunas pequeñas propiedades lograron realizarla y se dedicaron a la exportación. Véase: RoselaAr, Saskia, Public Land in the Roman Republic, cit. (n. 13), pp. 155-156.

${ }^{38}$ KaY, Philip, Rome's Economic Revolution (Oxford, Oxford University Press, 2014), pp. 87-106.

${ }^{39}$ App. BC 1,1,7,18: "oi galr plou/sioi th=sde th=j a)nemh/tou gh=j th $\backslash n$ pollh $\backslash n$ katalabo/ ntej kaiì $x r o / n \%$ qarrou=ntej ouÃ tina $s f a=j$ eÂti a)fairh/sesqai ta/ te a)gxou=sfįsin oÀsa te

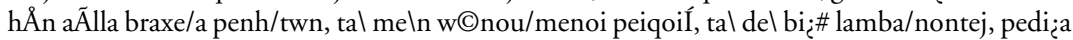

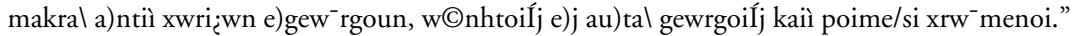

${ }^{40}$ App. BC 1,1,7,28: “a)pol del tou/twn oi ${ }_{s}$ meln dunatoiì pa/mpan e)plou/toun, kaiì tol tw ${ }^{1 / 2 n}$ qerapo/ntwn ge/noj a)na thln $\mathrm{xw}^{-}$ran e)plh/que, toulj d' ${ }^{1}$ Italiw ${ }^{-}$taj o)ligo/thj kaiì dusandrija katela/mbane, truxome/nouj peni_\# te kaì e)sforaiĺj kaì̀ stratei jaij”.

${ }^{41}$ BRUnT, Peter, The Army and the Land in the Roman Revolution, en Journal of Roman Studies, 52 (1962), pp. 69-86; y BRUNT, Peter, Fall of the Roman Republic and Related Essays (Oxford, Oxford University Press, 1988), p. 73.

${ }^{42}$ Rosenstein, Nathan, Rome at War (Chapel Hill - London, North Carolina University Press, 2004), pp. 26-62.
} 
duración indefinida. En efecto, hasta las guerras anibálicas las campañas militares solían desarrollarse en suelo italiano, en localidades relativamente cercanas al domicilio de los ciudadanos soldados que podían retornar a sus propiedades con el invierno y el fin de las hostilidades. La guerra era una actividad de corte estacional que no interfería con los ciclos agrícolas. Sin embargo, las campañas por la conquista de la cuenca del Mediterraneo supondrán el alejamiento permanente del campesino de su tierra por periodos indefinidamente largos para desarrollar operaciones en escenarios distantes. La conquista de Hispania supuso repetidas campañas y el estacionamiento de una fuerza de ocupación permanente. Otro tanto puede decirse de las guerras macedónicas y de las demás operaciones militares que terminaron por convertir el Mediterráneo en el Mare nostrum. Este tipo de guerra era incompatible con la economía agrícola del ciudadano campesino y sus resultados son la ruina de buena parte de esta clase media de la Antigüedad.

Una cruda descripción del estado de cosas nos la lega Plutarco ${ }^{43}$ : “Desposeídos los pobres, no se enrolaban en los ejércitos, ni alimentaban a los niños, estaba en riesgo Italia de quedarse completamente desierta de población libre y llenarse de prisioneros bárbaros, porque ellos labraban las tierras de los ricos, excluidos los ciudadanos".

Aunque hubo diversas tentativas de realizar una reforma agraria que revirtiese los desastrosos resultados de la expansión militar romana, ninguna de ellas consiguió restablecer a esa vieja clase de campesinos soldados que habían formado la columna vertebral del sistema republicano. La necesidad de reformas se hace evidente durante la segunda parte del siglo II a. C., cuando Cayo Lelio ${ }^{44}$ las promueve por primera vez. Sin embargo debió retirarlas ante la oposición senatorial. Mucho más importantes fueron las reformas llevadas adelante por los hermanos Tiberio y Cayo Graco que pretendían efectivamente reconstruir la clase media romana a fin de restablecer su poderío militar. Éstas se implementaron incluso ante la respuesta violenta por parte de la elite, aunque no fueron capaces de revertir la decadencia del pequeño campesino romano. De ahí en adelante, la demanda por reformas agrarias estuvo en el corazón de cada uno de los movimientos de corte revolucionario que golpearon la República hasta su final desarticulación por parte de Augusto y transformación en el Principado ${ }^{45}$.

El resultado fue el desplazamiento de los pequeños agricultores hacia la ciudad de Roma, la cual experimentó un vertiginoso incremento de su población que recuerda a las ciudades Latinoamericanas durante el siglo XX. La ciudad de Roma pasó de tener una población de entre 200 y 300 mil habitantes durante el siglo III a. C., a elevarse por sobre el millón hacia el fin de la República. Como una suerte de Valparaíso de la Antigüedad, la Roma tardo-republicana era una ciudad con

${ }^{43}$ PluT., Gracch. 8,4-5: “e)cwsqe/ntej oi, pe/nhtej ouÂte taiĺj stratei jaij eÂti proqu/mouj

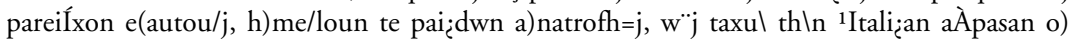

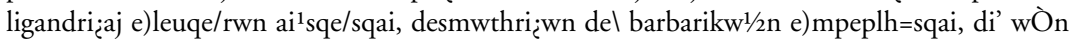
e)gew ${ }^{-}$rgoun oi , plou/sioi tal xwrįa, toulj poli ‘taj e)cela/santej”.

${ }^{44}$ Plut., Gracch. 8,5.

${ }^{45}$ Brunt, Peter, The Army and the Land in the Roman Revolution, cit. (n. 41), pp. 69-86. 
bolsones de miseria, donde los pobres vivían en barrios insalubres que colgaban de sus cerros, manteniéndose en constante peligro de incendio.

Resulta casi imposible cuantificar los resultados de este proceso y mensurar de alguna manera el retroceso de las clases medias en Roma tardo-republicana. No obstante, los efectos de dicha crisis social pueden, en cierta medida, apreciarse en razón de la ausencia de hombres elegibles para el servicio militar. Con una carga bélica bastante inferior a las veinticinco legiones que se llegaron a levar en las guerras anibálicas y con una población sensiblemente superior, Roma era simplemente incapaz de realizar la leva con éxito y llenar las legiones. En efecto, sólo con la reforma militar de Mario, que admitió por primera vez a los proletarios en él ${ }^{46}$, pudo realizarse la leva. De aquí en adelante las legiones las llenarán principalmente los ciudadanos más pobres de Roma, quienes verán en el ejército una salida a su miseria y en el éxito personal de los generales que los comandan, su única posibilidad de asegurarse su subsistencia.

Durante el siglo II a. C. Roma puso en pie entre ocho y doce legiones cada año, es decir, entre cuarenta y sesenta mil hombres ${ }^{47}$. La población de ciudadanos había aumentado sensiblemente, llegando hasta los casi cuatrocientos mil hombres mayores de edad ${ }^{48}$. Si realizamos el mismo cálculo compuesto anteriormente ${ }^{49}$ para las guerras anibálicas, llegamos a la conclusión que Roma antes de la reforma de Mario no era capaz de soportar una carga militar que supusiese la existencia de entre un 15 y un $18 \%$ de propietarios.

El resultado es elocuente. Aunque las cifras que entregamos no son del todo confiables y están afectas a variados caveat, sí corroboran el orden de magnitud del retroceso de lo que llamamos clase media, que pasa a comprender alrededor de dos tercios de la población a menos de un quinto. La crisis de gobernanza fue ineludible y la destrucción del sistema Republicano inevitable.

\section{CONCLUSIONES}

Las conclusiones que podemos obtener de este estudio son, en alguna media, una confirmación de la ya citada teoría de North. Los costos de mantener un sistema político son inversamente proporcionales a su legitimidad. La viabilidad de un sistema político participativo está directamente relacionada con los beneficios directos que la población obtenga de él. Si este tiende a la creación de una clase media amplia, favorecida por las oportunidades que la sociedad crea, el sistema se afianzará y consolidará. Si, en lugar de ello, reduce las oportunidades reales

${ }^{46}$ Plut., Marius 9.

${ }^{47}$ Seguimos la tabla realizada por Rosenstein, Nathan, Rome at War, cit. (n. 31), pp. 120-121).

${ }^{48}$ El resumen de Liv., Perioch. 63, da cuenta de 394.336 ciudadanos.

${ }^{49}$ Es decir, tomando la cifra máxima de levados $(60 \mathrm{mil})$, la población de assidui o propietarios necesaria para llenar la leva sería de 240 mil personas. Mientras que agregando a los 400 mil hombres mayores de 14 años la proporción faltante de mujeres y niños, llegaríamos a una cifra total de población ciudadana que fluctúa entre 1,3 y 1,6 millones de ciudadanos, según la esperanza de vida al nacer que estimemos para Roma republicana. Esto daría entre un 18 y $15 \%$ de propietarios sobre el censo mínimo. 
de avance personal y pauperiza a sus sectores medios, entrará inevitablemente en crisis y terminará por sucumbir ante las ambiciones de elementos desestabilizadores que buscarán reemplazarlo, sea por uno que tienda a la efectiva distribución de la riqueza, sea por uno que fundamente su estabilidad en un uso más profundo de la coacción como herramienta de control social.

En el caso romano, fue el Principado lo que nació de las cenizas de la República, un orden político intermedio que tendió a favorecer el comercio y la estabilidad social, aunque el costo fue que el sistema dejó de ser participativo con toda claridad. Efectivamente, el nivel de coacción aplicado para mantener el sistema político fue mayor que en la República media, aunque inferior a los niveles alcanzados por los despotismos tardíos.

La caída de lo que hemos denominado clase media romana se produjo en un período de tiempo relativamente corto, alrededor de cien años, aunque sus primeros efectos eran visibles antes.

\section{BiBLIOGRAFÍA}

Arangio-Ruiz, Vincenzo, Storia del diritto romano (Napoli, Jovene, 2006).

Bonfante, Pietro, La gens e la familia, en Scritti giridici. Famiglia e successione (Torino, Unione Tipografico, 1916).

Bonfante, Pietro, Teorie vecchie e nuove sulle formazione sociali primitive en Scritti giuridici. Famiglia e successione (Torino, Unione Tipografico, 1916).

BRUnT, Peter, Fall of the Roman Republic and Related Essays (Oxford, Oxford University Press, 1988).

Brunt, Peter, Italian Manpower (London, Oxford University Press, 1971).

Brunt, Peter, The Army and the Land in the Roman Revolution, en Journal of Roman Studies, 52 (1962).

Capogrossi Colognesi, Luigi, Diritto e potere nella storia di Roma (Napoli, Jovene, 2007).

Capogrossi Colognesi, Luigi, La terra in Roma antica (Roma, La Sapienza, 1981).

Castello, Carlo, Studi sul diritto familiar e gentilizio romano (Roma, L'Erma di Bretschneider, 1942).

Cornell, Tim, The Beginnings of Rome and Italy from the Bronze Age to the Punic Wars (c. 1000-264 BC), (London - New York, Routledge, 1995).

Cornell, Tim, The Conquest of Italy, en Walbank, F. W. - Austin, A. E. - Frederiksen, M. W. - Ogilvie, R. M. (editores), The Cambridge Ancient History (Cambridge, Cambridge University Press, 1989), VII, 2.

De Martino, Francesco, Clienti e condizioni materiali in Roma arcaica, en Diritto, economia e società nel mondo romano (Napoli, Jovene, 1997).

De Martino, Francesco, Intorno all origine della schiavitù a Roma, en Diritto, economía e società nel mondo romano (Napoli, Jovene, 1997).

De Martino, Francesco, La gens, lo stato e le classi in Roma antica, en Diritto, economía e società nel mondo romano (Napoli, Jovene, 1997).

De Martino, Francesco, Storia costituzionale romana (Napoli, Jovene, 1973).

Drummond, Andrew, Rome in the Fifth Century, II: The Citizen Community, en Walbank, F. W. - Austin, A. E. - Frederiksen, M. W. - Ogilvie, R. M. (editores), The Cambridge Ancient History (Cambridge, Cambridge University Press, 1989), VII,2. 
Forsythe, Gary, A Critical History of Early Rome (Berkeley - Los Ángeles, California University Press, 2006).

Franciosi, Gennaro, "Res mancipi" e "res nec mancipi", en Labeo 5 (1959) 3.

Franciosi, Gennaro, Famiglia e persone in Roma antica (Torino, Giappichelli,1992).

GABBA, Emilio, Motivazioni economiche nell opposizione alla lege agraria di Tib. Sempronio Graccho, en Evans, J. A. S., "Polis" and "Imperium": Studies in Honour of Edward Togo Salmon (Toronto, Hakkert, 1974).

Guarino, Antonio, Forma e materia della costituzione romana, en Studi di diritto costituzionale romano (Napoli, Jovene, 2008).

GuARINO, Antonio, Le origine quiritarie. Raccolta di scritti romanistici (Napoli, Jovene, 1973).

Harmand, Louis, Société et économie de la république romaine (Paris, Armand Colin, 1976).

Harris, William, On the Applicability of the Concept of Class in Roman History, en Rome's Imperial Economy. Twelve Essays (Oxford - New York, Oxford University Press - Kindle, 2011).

Harris, William, Poverty and Destitution in the Roman Empire en Rome's Imperial Economy, Twelve Essays (Oxford - New York, Oxford University Press - Kindle, 2011).

Harris, William, The Late Republic, en Scheidel, Walter - Morris, Ian - Saller, Richard (editores), The Cambridge Economic History of the Greco-Roman World (Cambridge, Cambridge University Press, 2007).

KaY, Philip, Rome's Economic Revolution (Oxford, Oxford University Press, 2014).

Kehoe, Denis, Contract labor, en Scheidel, Walter (editor), Roman Economy (New York, Cambridge University Press, 2012).

Knapp, Robert, Invisible Romans (Cambridge, Harvard University Press, 2011).

Lo Cascio, Elio, Population and Demographic Studies, en Derose Evans, Jane (editora), A Companion to the Archeology of the Roman Republic (Oxford, Blackwell, 2013).

Magdelain, André, De L'auctoritas patrum à Pauctoritas senatus en Jus imperium auctoritas. Etudes de droit romain (Paris, Ecole Française de Rome, 1990).

Manzo, Annamaria.. La lex licinia sextia de modo agrorum (Napoli, Jovene, 2001).

MaschKe, Richard, Zur Theorie und Geschichte der römischen Agrargesetze (Napoli, Jovene, 1980).

Mitchell, Richard, The Definition of "patres" and "plebs": An End to the Struggle of the Orders, en RaAflaub, Kurt (editor), Social Struggles in Archaic Rome. New Prespectives on the Conflict of the Orders (Oxford, Blackwell, 2005).

Momigliano, Arnaldo, The Origins of Rome, en Walbank, F. W. - Austin, A. E. Frederiksen, M. W. - Ogilvie, R. M. (editores), The Cambridge Ancient History (Cambridge, Cambridge University Press, 1989), VII,2.

Momigliano, Arnaldo, The Rise of the plebs in the Archaic Age of Rome, en RaAflaub, Kurt (editor), Social Struggles in Archaic Rome. New Prespectives on the Conflict of the Orders (Oxford, Blackwell, 2005).

Morley, Neville, Trade in Classical Antiquity (Cambridge, Cambridge University Press - Kindle, 2007).

NorTH, Douglas, Structure and Change in Economic History (New York, Norton, 1981). PIKETTY, Thomas, Capital in the Twenty-First Century, (Cambridge, Harvard University Press- Kindle, 2014).

Raaflaub, Kurt A., From Protection and Defense to Offense and Participation: Stages in 
the Conflict of the Orders, en RaAflaub, Kurt (editor), Social Struggles in Archaic Rome. New Prespectives on the Conflict of the Orders (Oxford, Blackwell, 2005).

Raaflaub, Kurt, The Conflict of the Orders in Archaic Rome: A Comprehensive and

Comparative Approach, en RaAflaub, Kurt (editor), Social Struggles in Archaic Rome. New Prespectives on the Conflict of the Orders (Oxford, Blackwell, 2005).

Richard, Jean-Claude, Patricians and Plebeians: The Origin of a Social Dichotomy, en RaAflaub, Kurt (editor), Social Struggles in Archaic Rome. New Prespectives on the Conflict of the Orders (Oxford: Blackwell, 2005).

RicharD, Jean-Claude, Réflexions sur les origines de la plebe en Bilancio critico su Roma arcaica fra monarchia e repubblica (Roma, Academia Nazionale dei Lincei, 1993).

Roberts, Keith, Origins of Business, Money and Markets (New York, Columbia University Press - Kinlde, 2011).

Roselaar, Saskia, Public Land in the Roman Republic. A Social and Economic History of "Ager Publicus" in Italy, 396-89 BC (Oxford - New York, Oxford, 2010).

RosensteIn, Nathan, Rome at War (Chapel Hill - London, North Carolina University Press, 2004).

Russo, Adelaide, Tiberio Gracco e la riforma agraria, en La romanizzazione della Campania antica (Napoli, Jovene, 2002).

Saller, Richard P., Patriarchy, Property and Death in the Roman Family (Cambridge, Cambridge University Press, 1994).

SALLER, Richard, Framing the Debate over Growth in the Ancient Economy en SCHEIDEL, Walter - Von Reden, Sitta (editores), The Ancient Economy (New York, Routledge, 2010).

Scheidel, Walter, Demography, en Scheidel, Walter - Morris, Ian - SAlLer, Richard (editores), The Cambridge Economic History of the Greco-Roman World (Cambridge, Cambridge University Press, 2007).

SERRAO, Feliciano, Lotte per la terra e per la casa a Roma, en SERRAO, Feliciano (editor), Legge e società nella repubblica romana (Napoli, Jovene, 1981).

SMITH, Christopher, The Roman Clan. The gens from Ancient Ideology to Modern Anthropology (Cambridge, Cambridge University Press, 2006).

Temin, Peter, The Roman Market Economy (New Jersey, Princeton University Press, 2013).

Terrenato, Nicola, The Essential Countryside, en Alcock, Susan - Osborne, Robin (editores), Classical Archeology (Malden - Oxford, Blackwell, 2012).

Torelli, Mario, History: Land and People, en Bonfante, Larissa (editora), Etruscan Life and Afterlife, (Detroit, Wayne State University Press, 1986). 